\title{
Uso de servicios de planificación familiar de la Secretaría de Salud, poder de decisión de la mujer y apoyo de la pareja
}

\author{
Fátima Estrada, MSc, (I) Carlos Hernández-Girón, MD, ${ }^{(I)}$ Dilys Walker, MD, (I) Lourdes Campero, MSc, ${ }^{(I)}$ \\ Bernardo Hernández-Prado, PhD, (I) Catherine Maternowska, PhD. ${ }^{(2)}$
}

\begin{abstract}
Estrada F, Hernández-Girón C,Walker D, Campero L, Hernández-Prado B, Maternowska C. Uso de servicios de planificación familiar de la Secretaría de Salud, poder de decisión de la mujer y apoyo de la pareja. Salud Publica Mex 2008;50:472-48I.
\end{abstract}

\section{Resumen}

Objetivo: Identificar la influencia de las características reproductivas y el empoderamiento femenino en el uso de servicios de planificación familiar (PF). Material y métodos: Diseño de casos y no casos pareado por controles vecindarios (I47 usuarias y |46 no usuarias de servicios de PF de la Secretaría de Salud durante 2003), en dos municipios del estado de Guanajuato, México. Análisis logístico multivariado para identificar diferencias entre usuarias y no usuarias. Resultados: El uso de servicios de PF se asoció positiva y significativamente con poder de decisión de la mujer (alto: $R M=3.2$; IC95\% I.4-7.4); comunicación con la pareja para el uso de métodos de PF (RM =3.5; IC95\% I.4-9.3), y número de embarazos en su vida (>6 hijos: $R M=4.4 ; \mid C 95 \%$ I.4- $\mid 3.8$ ). Conclusiones: El desarrollo de estrategias que involucren a los hombres y que, por otra parte, fomenten y fortalezcan el empoderamiento femenino, puede contribuir a una mayor utilización de servicios de PF.

Palabras clave: servicios de planificación familiar; poder; género y salud; México
Estrada F, Hernández-Girón C,Walker D, Campero L, Hernández-Prado B, Maternowska C.

Use of family planning services and its

relationship with women's decision-making

and support from their partner.

Salud Publica Mex 2008;50:472-48I.

\section{Abstract}

Objective. To identify the influence of reproductive characteristics and women empowerment with the use of family planning services. Material and Methods. Cases and noncases design with neighborhood controls (I47 users and I46 non-users of family planning services during 2003), in Guanajuato State, Mexico.Various indexes were constructed to evaluate women's empowerment and its relationship with family planning use. Results. The use of family planning services was positively and significantly associated with the woman's power to make decisions (High: OR 3.2, CI95\% I.4-7.4), a high level of communication with her partner on contraceptive use (OR 3.5, CI95\% I.4-9.3); and a greater number of pregnancies (> 6 children: OR 4.4, CI95\% I.4- I3.8). Conclusion. Factors such as a high level of female decisionmaking and more partner support for contraceptive use are related to the use of family planning services. Therefore, developing strategies that involve men and support female empowerment could contribute to increasing the use of family planning services.

Key words: family planning services; power; gender and health; Mexico

(I) Centro de Investigaciones en Salud Poblacional. Instituto Nacional de Salud Pública. Cuernavaca, Morelos, México.

(2) Center for Reproductive Health and Policy, University of California. San Francisco, California, USA.

Fecha de recibido: 7 de septiembre de 2007 - Fecha de aceptado: 14 de mayo de 2008 Solicitud de sobretiros: MD. Carlos Hernández Girón. Centro de Investigaciones en Salud Poblacional, Instituto Nacional de Salud Pública. Avenida Universidad 655, col. Santa María Ahuacatitlán. 62508, Cuernavaca, Morelos, México. Correo electrónico: chernand@correo.insp.mx 
L planificación familiar (PF) contribuye a salvar vidas de mujeres y niños y mejora la calidad de vida de todos. De hecho, es una de las mejores inversiones posibles para mantener la salud y el bienestar de las mujeres, los niños y las comunidades. ${ }^{1} \mathrm{El}$ hecho de contar y de acudir a servicios de planificación familiar (PF) le permite a las parejas elegir, de manera informada, el método anticonceptivo que mejor se adapte a sus necesidades para controlar su fecundidad y el espaciamiento de los embarazos. ${ }^{2}$

En México han ocurrido importantes cambios en el plano nacional a este respecto, pues el uso de métodos anticonceptivos en mujeres unidas pasó de $30.2 \%$ en 1976 a $70.8 \%$ en $2000 .{ }^{3,4}$ Específicamente en el estado de Guanajuato, la proporción de uso de métodos fue de $59.1 \%$ para 2000, es decir, inferior a la media nacional. Esta relación se presenta de la misma manera para el caso de la tasa de fecundidad; mientras que en todo el país, de 1990 a 2000, descendió de 3.4 a 2.4, en Guanajuato descendió de 3.7 a 2.8 . $^{3}$

La falta de información, o bien, la adquisición de conocimientos erróneos sobre la existencia de anticonceptivos, así como la ausencia de asesoría, la consejería deficiente, la postura de algunas religiones, las barreras para conseguir los métodos anticonceptivos por inexistencia de servicios, los costos, las diferencias culturales y lingüísticas y la inequidad de género representan factores particularmente importantes para el uso adecuado de anticonceptivos. ${ }^{5}$

La inequidad de género en el ámbito de la salud se refiere a las desigualdades injustas, innecesarias y prevenibles que existen entre las mujeres y los hombres en el estado y la atención de salud. ${ }^{6}$ La forma de medirlas es una de las mayores dificultades que se presentan al investigar la inequidad de poder basada en el género. Desde la década de 1990 se han desarrollado escalas para cuantificar las relaciones de poder en las parejas; los instrumentos se basan en preguntas acerca de quién toma las decisiones, quién tiene la última palabra en decisiones específicas, en el control sobre los ingresos, y/o la libertad de movimiento..$^{7-9}$ Una aproximación integral para la medición de poder dentro de la pareja es la Escala de Poder en las Relaciones Sexuales (Sexual Relationship Power Scale, SRPS por sus siglas en inglés) que desarrollaron Pulerwitz y colaboradores en 2000. ${ }^{10}$

Diversas investigaciones han mostrado que el uso de métodos anticonceptivos, el número ideal de hijos, y la negociación de uso del condón están estrechamente ligados a la relación de género en la pareja. ${ }^{11-16}$ Sin embargo, poco se ha escrito sobre la forma en que las relaciones de género en la pareja influyen al acudir a los servicios de planificación familiar.
En 2003, la Universidad de California, San Francisco, en los Estados Unidos de América (EUA), y el Instituto Nacional de Salud Pública, en Cuernavaca, Morelos, México, llevaron a cabo una investigación con enfoque cuali-cuantitativo para identificar la diferencia en las características de salud reproductiva, con especial énfasis en las percepciones e ideas sobre las relaciones de género dentro de la pareja y el empoderamiento femenino, entre las mujeres de bajo nivel socioeconómico que acuden a servicios de planificación, a diferencia de aquellas que no lo hacen. Este artículo presenta los resultados del componente cuantitativo de este estudio.

\section{Material y métodos}

Durante el segundo semestre de 2003, se realizó un estudio de casos y no casos, retrospectivo, pareado por edad y controles vecindarios en mujeres en edad reproductiva residentes del estado de Guanajuato, México. La recolección de información se llevó a cabo en dos municipios, y en cada uno se incluyó una localidad urbana -conformada por la cabecera de cada municipio, por contar con una población de más de 2500 habitantes-, y dos rurales-seleccionadas aleatoriamente entre aquellas que contaban con menos de 2500 habitantes, de acuerdo con el Conteo de Población y Vivienda 1995. ${ }^{17}$

Los criterios de elegibilidad para ambos grupos fueron ser mujer mexicana residente de los sitios previamente seleccionados, con edad entre 18 y 49 años, con posibilidad de embarazo, sin ningún tipo de seguro médico público o privado, y de ingreso socioeconómico bajo.

Los casos fueron definidos como usuarias de los servicios de PF de la Secretaría de Salud (SSA) en el último año y los no casos como no usuarias de los servicios de PF de la SSA en los últimos tres años.

Los casos fueron reclutados en clínicas de la SSA, ya que esta institución atiende a población abierta, es decir, a personas que no están afiliadas a alguna institución de seguridad social. En el estado de Guanajuato la SSA brinda servicios en los niveles primario y secundario a través de 474 clínicas y consultorios. ${ }^{18}$

Para la selección de los casos en las clínicas de las zonas urbanas, a cada tercer mujer que ingresaba en la clínica se le aplicaba el cuestionario de elegibilidad, y en las clínicas rurales se aplicó este cuestionario a todas las mujeres que ingresaban. Una vez que se definía si eran elegibles, se les invitaba a participar en el estudio, aplicando la encuesta a aquellas que aceptaban y habían firmado la carta de consentimiento informado. Se estimó un tamaño de muestra de 586 sujetos. 
Los no casos se seleccionaron aleatoriamente en las zonas urbanas de los mismos vecindarios que los casos, y en las zonas rurales de las mismas comunidades.

Se aplicó un cuestionario de 154 preguntas, dividido en las siguientes secciones: I. Características sociodemográficas; II. Apoyo y relaciones sociales; III. Relaciones personales; IV. Dinámica de la relación de pareja; V. Actividad sexual; VI. Normas culturales; VII. Anticoncepción; VIII. Uso de los servicios de salud reproductiva, y IX. Experiencia migratoria.

Los reactivos de la sección IV se tomaron de la escala SRPS, que se desarrolló con información recolectada mayoritariamente de mujeres latinas en clínicas de los EUA. Esta escala consta de 23 reactivos y está dividida en dos subescalas: la correspondiente al control de la relación y la vinculada con el dominio de la toma de decisiones. La escala se validó tanto en inglés como en español; la confiabilidad de la versión en español de toda la escala fue de .88 , y, de las subescalas, de $.89 \mathrm{y}$ .60 respectivamente. ${ }^{10}$

Con las preguntas de la escala SRPS se construyeron cinco índices para evaluar algunas características del empoderamiento femenino (cuadro I):

\section{Cuadro I \\ Preguntas utilizadas para la construcción de índices de Relaciones de PODER en USUARIAS Y No USUARIAS de los Servicios de planificación familiar de la SSA en Guanajuato, México, 2003}

Índice

$\begin{array}{cc}\text { Opciones } & \text { Alfa } \\ \text { de respuesta } & \text { Ponderador de Cronbach }\end{array}$

I. Poder de decisión:

I. ¿La opinión de quién tiene mayor peso para las pequeñas compras diarias del hogar (como abarrotes o ropa)?

2. La opinión de quién tiene mayor peso para las grandes compras para la casa (como una televisión o un refrigerador)?

3. Cuando están juntos, iquién tiene mayor peso para decidir qué hacer juntos?

4. ¿La opinión de quién tiene mayor peso para escoger el momento para hablar de cosas serias?

5. ¿La opinión de quién tiene mayor peso para decidir que usted use un método anticonceptivo?

6. ¿La opinión de quién tiene mayor peso para decidir cuándo y cuántos hijos tener?

7. En general, iquién cree usted que manda en su relación?

2. Apoyo para el uso de anticonceptivos:

I. Si usted le dijera a él de repente que usted está usando un método anticonceptivo, ¿[NOMBRE] desconfiaría de usted?

2. Si alguna vez usted le pidiera que usara un condón, ise enojaría [NOMBRE]?

3. Si alguna vez usted le pidiera que usara un condón, ise pondría violento [NOMBRE]?

3. Índice de rechazo a la violencia:

¿Cree usted que si una mujer se niega a tener relaciones sexuales con su pareja cuando él quiere, él tiene derecho a enojarse y regañarla?

2. ¿Cree usted que si una mujer se niega a tener relaciones sexuales con su pareja cuando él quiere, él tiene derecho a negarse a darle dinero (u otros medios de apoyo financiero)?

3. ¿Cree usted que si una mujer se niega a tener relaciones sexuales con su pareja cuando él quiere, él tiene derecho a pegarle o darle una golpiza?

4. ¿Cree usted que si una mujer se niega a tener relaciones sexuales con su pareja cuando él quiere, él tiene derecho a forzarla a tener relaciones sexuales aunque ella no quiera?

5. ¿Cree usted que si una mujer se niega a tener relaciones sexuales con su pareja cuando él quiere, él tiene derecho a tener relaciones sexuales con otra mujer?

4. Equidad de roles de género:

I. Según la gente en su comunidad, ¿es importante que la mujer se embarace lo más pronto posible después de casarse?

2. Según la gente en su comunidad, ¿quién debe mandar en una relación?

3. Según la gente en su comunidad, ¿quién debe controlar el dinero?

$\begin{array}{cr}\text { Muy frecuente: } 0 & 0.40 \\ \text { No muy frecuente: } & 0.60\end{array}$

Nunca: 2

Ambos:

Ella: 2

0.34

0.66

Autonomía en las relaciones sexuales:

I. ¿Cree usted que la mujer tiene razón en negarse a tener relaciones sexuales con su pareja porque está cansada y no tiene ganas?

2. ¿Cree usted que la mujer tiene razón en negarse a tener relaciones sexuales con su pareja porque acaba de dar a luz?

3. ¿Cree usted que la mujer tiene razón en negarse a tener relaciones sexuales con su pareja porque ella sabe que su pareja tiene relaciones sexuales con otras mujeres?

4. ¿Cree usted que la mujer tiene razón en negarse a tener relaciones sexuales con su pareja porque ella sabe que su pareja tiene una enfermedad transmitida sexualmente?

Fuente: Índices desarrollados a partir de los reactivos tomados de la Sexual Relationship Power Scale, desarrollada por Pulerwitz y colaboradores en $2000 .{ }^{10}$ 
1. Poder de decisión de la mujer: percepción sobre el grado de negociación con la pareja sobre situaciones cotidianas y decisiones reproductivas.

2. Apoyo para uso de anticonceptivos: percepción del grado de negociación con la pareja sobre el uso de anticonceptivos.

3. Rechazo a la violencia: ideas sobre el grado de aceptación o rechazo respecto a acciones violentas por parte del hombre hacia la mujer.

4. Equidad de roles de género: ideas sobre la igualdad entre el hombre y la mujer en una relación de pareja.

5. Autonomía en las relaciones sexuales: ideas sobre el grado de negociación entre hombre y mujer en las relaciones sexuales.

Se realizó un análisis de componentes principales para identificar la contribución de cada reactivo a la variabilidad general de cada uno de los índices, siguiendo la metodología propuesta por Cacique. ${ }^{13}$ Este análisis mostró que las preguntas contribuían de manera distinta a la variabilidad de cada índice, por lo que se obtuvieron los ponderadores de cada pregunta a partir del análisis de componentes principales, y se optó por trabajar en el análisis con preguntas ponderadas en la construcción de los índices. Asimismo, se calculó el alfa de Cronbach, para evaluar el grado de consistencia interna de las preguntas consideradas en cada índice. Con este procedimiento se obtuvo una escala continua en cada uno de los índices. Más adelante, una vez evaluada su distribución de normalidad mediante la evaluación de gráficas, se procedió a categorizarlos con base en terciles, en tres grados: bajo, medio y alto.

El análisis estadístico comprendió una fase descriptiva, con la obtención de frecuencias, medidas de tendencia central y dispersión de las variables de interés. Mediante la obtención de razones de momios $(R M)$ y sus respectivos intervalos de confianza al 95\% (IC95\%), se estimó la relación entre los índices del empoderamiento femenino y el uso de servicios de PF en la SSA, con modelos de regresión logística condicional. De igual manera, se ajustó un modelo de regresión logística condicional multivariado, donde se incluyó como variable dependiente el uso de servicios de PF de la SSA en el último año, y como variables independientes la edad, la escolaridad, la edad de inicio de relaciones sexuales, los antecedentes obstétricos, el uso de condón masculino alguna vez, el índice de poder de decisión, el de apoyo para uso de métodos de PF, el de rechazo a la violencia, el de equidad de género, y el de autonomía en las relaciones sexuales. La información se analizó mediante el paquete estadístico Stata 8.0 (Stata Corporation. Stata reference Manual: Release 8.0. College Station Tx USA, 2002).

Este proyecto fue aprobado por las comisiones de ética, bioseguridad e investigación, tanto de la Universidad de California, San Francisco, EUA, como del Instituto Nacional de Salud Pública.

\section{Resultados}

Para este estudio se estableció contacto con un total de 586 mujeres, considerando los criterios de elegibilidad; se obtuvo una tasa de participación de $97 \%$ en el grupo de las usuarias y de $91 \%$ para las no usuarias, quedando al final una muestra de 500 mujeres, de las cuales 250 eran casos y 250 no casos. Para el análisis, se eliminaron aquellos cuestionarios que no tenían la información completa en las variables de interés (98 casos y 86 no casos), así como aquellas mujeres que refirieron no ser sexualmente activas al momento de la encuesta (un caso y cuatro no casos), y aquellas que estaban lactando (cuatro casos y 14 no casos). Después de eliminar estas observaciones, se llevó a cabo el análisis con 293 observaciones (147 casos y 146 controles). Las mujeres con información incompleta no difirieron en sus características demográficas de aquellas con información completa.

\section{Características sociodemográficas}

El promedio de edad fue de $28.5 \pm 5.45$ años para las usuarias y de $28.1 \pm 5.69$ años para las no usuarias de los servicios de PF de la SSA ( $\mathrm{p}>0.05)$. La mayor proporción de mujeres en ambos grupos, usuarias (96\%) y no usuarias $(97 \%)$, correspondió a mujeres casadas o que vivían en unión libre con su pareja $(p>0.05)$ (cuadro II).

Con respecto al grado de escolaridad, ambos grupos tuvieron el mayor porcentaje de mujeres con nivel primaria; sin embargo un mayor número de no usuarias $(24 \%)$ refirieron no contar con educación formal, a diferencia de las usuarias (16\%), y esta relación se invirtió en el caso de la secundaria, donde se encontró un mayor porcentaje en la usuarias $(21 \%)$ que en las no usuarias (17\%), aunque estas diferencias no fueron estadísticamente significativas.

La mayoría de las entrevistadas tanto en el grupo de las usuarias como de las no usuarias, refirieron dedicarse a actividades relacionadas con el hogar. La religión predominante fue la católica (cuadro II). En relación con el lugar de residencia, la distribución en el grupo de usuarias y no usuarias fue la misma; $64 \%$ correspondían a zona urbana y $36 \%$ a zona rural. 
Cuadro II

Características Sociodemográficas en usuarias

Y NO USUARIAS DE LOS SERVICIOS DE PLANIFICACIÓN familiar de la SSA en Guanajuato, México, 2003

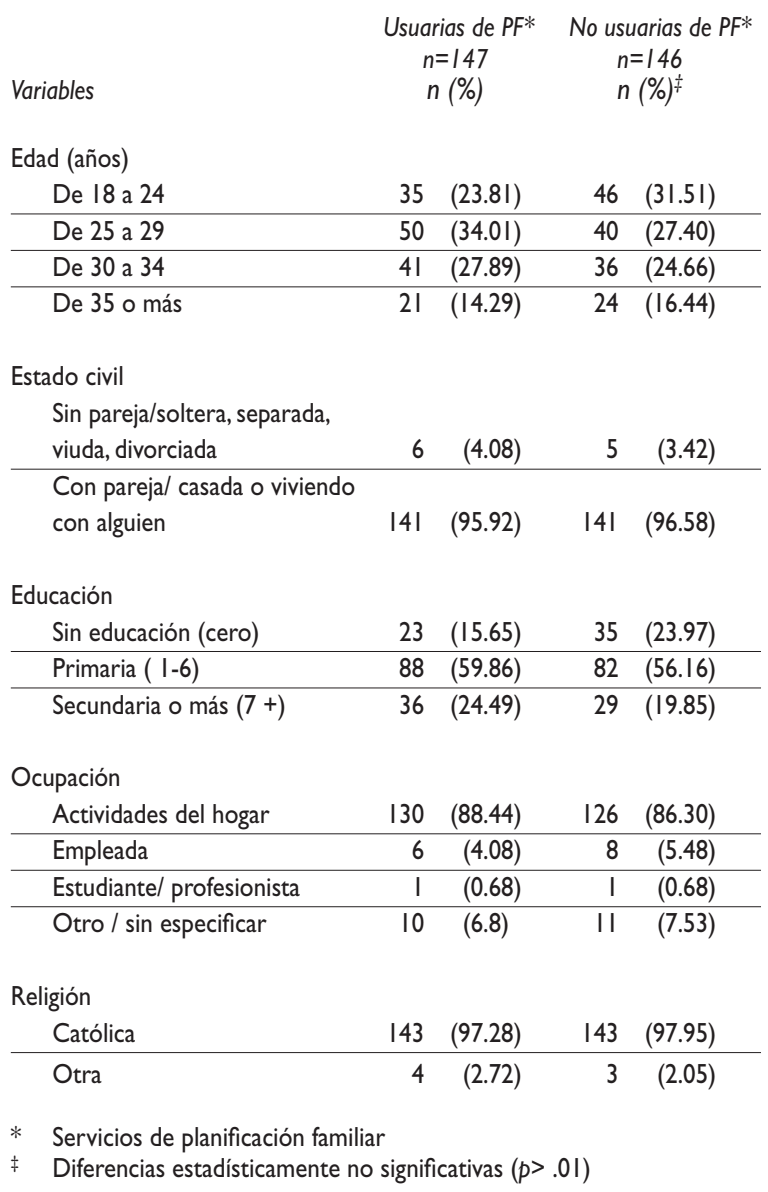

Fuente: a partir de datos recogidos en el proyecto "Género, poder, cultura y salud reproductiva en México”: agosto-noviembre 2003

\section{Comportamiento sexual, uso de métodos anticonceptivos y características obstétricas}

El promedio de edad de inicio de vida sexual de las usuarias de servicios fue de 18.2 años ( $D E \pm 3.18$ ), y en el grupo de mujeres no usuarias de 18.5 años $(D E \pm 3.12)$ ( $p>0.05)$; más de $90 \%$ de las mujeres de ambos grupos vivieron esta primera relación sexual como voluntaria. Por otra parte, más de la mitad de las entrevistadas en ambos grupos refirieron una frecuencia de relaciones sexuales superior a dos veces por semana (cuadro III).

Con respecto a la última relación sexual, $53 \%$ de las entrevistadas $-85 \%$ de las usuarias y $21 \%$ de las no usuarias $(p<0.05)$ - refirieron haber utilizado algún método anticonceptivo. Entre las usuarias de servicios, $56 \%$ notificaron usar anticonceptivos hormonales o DIU, a diferencia de sólo $2 \%$ de las no usuarias de servicios. Del total de las participantes, $82 \%$ notificaron el uso del condón masculino, con su pareja sexual, alguna vez en su vida; esta proporción fue mayor entre las usuarias de servicios $(88.4 \%)$ que entre las no usuarias $(74 \%)(p<0.05)$. Por otra parte, sólo $2 \%$ del total de entrevistadas refirieron haber usado alguna vez el método de anticoncepción de emergencia; las seis mujeres que lo notificaron pertenecían al grupo de usuarias de servicios de PF (figura I).

En lo tocante a las características obstétricas, el promedio de edad al primer parto en el grupo de usuarias de servicios fue de 19.5 años, y en el de no usuarias de 20 años ( $p>0.05$ ); el promedio de hijos vivos informados fue de 3.6 en las usuarias, y de 2.8 en las mujeres no usuarias $(p<0.05)$. Por otra parte, $22 \%$ refirieron el antecedente de aborto, siendo mayor esta proporción entre las no usuarias (27\%) que entre las usuarias (17\%) $(p<0.05)$; cabe mencionar que ninguna de las nulíparas refirió tener antecedente de aborto (cuadro III).

\section{Relaciones de género y utilización de servicios de planificación familiar}

Al indagar sobre características de uso de los servicios de $\mathrm{PF}$, se preguntó a las participantes si tenían comunicación con su pareja con respecto al uso de métodos anticonceptivos; $92 \%$ de las usuarias y $75 \%$ de las no usuarias respondieron afirmativamente. Asimismo, al preguntar si recibían apoyo de su pareja para el uso de algún método, 98\% de las usuarias y 79\% de las no usuarias refirieron contar con el apoyo de su pareja $(p<0.05)$ (cuadro III).

De los cinco índices que se construyeron para identificar las características de la relación de género dentro de la pareja, se encontraron diferencias estadísticamente significativas en dos de ellos: poder de decisión de la mujer y autonomía en las relaciones sexuales. Así, se observa un mayor grado en el índice de poder de decisión en las usuarias, comparado con las no usuarias de servicios de PF de la SSA (grado alto: $36 \%$ usuarias, $22 \%$ no usuarias) $(p<0.05)$. De igual manera, las mujeres usuarias mostraron en mayor proporción niveles más altos en el índice de autonomía en las relaciones sexuales (alto: 78.2\%), en comparación con las no usuarias (alto: $66.7 \%$ ). Los datos muestran una mayor proporción de apoyo para el uso de anticonceptivos por su pareja en las usuarias de servicios de PF (medio-alto: 90.4\%), comparado con las no usuarias (medio-alto: $87.8 \%$ ); sin embargo, estas diferencias no fueron estadísticamente significativas. Los dos índices correspondientes a rechazo a la violencia y equidad de género, no mostraron diferencias importantes por destacar (cuadro III). 


\section{Cuadro III}

\section{Características de comportamiento sexual y de Relaciones de poder en USUarias y No usuarias de los servicios de PLANificación familiar de la SSA en Guanajuato, MéXico, 2003}

Índice

Edad inicio vida sexual (años)

\begin{tabular}{|c|c|c|c|c|c|c|}
\hline$>20$ & 38 & 25.8 & 40 & 27.4 & 1.0 & - \\
\hline $15-19$ & 100 & 68.0 & 100 & 68.0 & I.I & $0.6-1.8$ \\
\hline$<15$ & 9 & 6.1 & 6 & 4.1 & 1.6 & $0.5-5.0$ \\
\hline \multicolumn{7}{|c|}{ Característica la. relación sexual } \\
\hline Voluntaria & 137 & 93.2 & 139 & 95.8 & 1.0 & - \\
\hline Involuntaria & 10 & 6.8 & 6 & 4.1 & 1.7 & $0.6-4.8$ \\
\hline
\end{tabular}

Frecuencia relaciones sexuales ${ }^{\ddagger}$

\begin{tabular}{|c|c|c|c|c|c|c|}
\hline$<3$ veces al mes & 29 & 20.5 & 28 & 20.2 & 1.0 & - \\
\hline $\mathrm{I}-2$ veces a la semana & 31 & 21.9 & 28 & 20.2 & 1.4 & $0.6-3.4$ \\
\hline$>2$ veces a la semana & 81 & 57.4 & 82 & 59.4 & 1.2 & $0.5-2.8$ \\
\hline
\end{tabular}

Número parejas sexuales ${ }^{\ddagger}$

\begin{tabular}{lrrrrrrr} 
Una & 141 & 95.9 & 145 & 99.3 & 1.0 & - \\
\hline$\geqq 2$ & 6 & 4.1 & 1 & 0.7 & 0.2 & $0.0-1.4$
\end{tabular}

Comunicación con pareja sobre métodos anticonceptivos

\begin{tabular}{lrrrrrrr} 
No & 12 & 8.2 & 36 & 24.7 & No & 12 \\
\hline Sí & 135 & 91.8 & 110 & 75.3 & Sí & 135
\end{tabular}

Apoyo de la pareja para uso de métodos anticonceptivos $\S$

\begin{tabular}{lrrrrrrr} 
No & 3 & 2.2 & 23 & No & 3 & 2.2 \\
\hline Sí & $|3|$ & 97.8 & 87 & Sí & $|3|$ & 97.8
\end{tabular}

Uso condón masculino alguna vez en su vida

\begin{tabular}{lrrrrrrr} 
No & 17 & 11.6 & 37 & 25.2 & No & 17 \\
\hline Sí & 130 & 88.4 & 110 & 74.8 & Sí & 130
\end{tabular}

Paridad

\begin{tabular}{|c|c|c|c|c|c|c|}
\hline $0-2$ & 54 & 38.1 & 64 & 43.5 & 1.0 & - \\
\hline $3-5$ & 66 & 44.9 & 66 & 44.9 & 1.3 & $0.8-2.3$ \\
\hline 6 y más & 27 & 18.4 & 17 & 11.6 & 2.1 & I.0-4.7 \\
\hline
\end{tabular}

Abortos previos

\begin{tabular}{lrrrrrrr} 
No & 122 & 83.0 & 107 & 72.8 & 1.0 & - \\
\hline Sí & 25 & 17.0 & 40 & 27.2 & 0.5 & $0.3-1.0$
\end{tabular}

Poder de decisión

\begin{tabular}{|c|c|c|c|c|c|c|}
\hline Bajo & 24 & 16.3 & 46 & 31.3 & 1.0 & - \\
\hline Medio & 70 & 47.6 & 68 & 46.3 & 1.8 & $1.0-3.4$ \\
\hline Alto & 53 & 36.1 & 33 & 22.4 & 2.9 & I.5-5.7 \\
\hline
\end{tabular}

Apoyo para uso de anticonceptivos

\begin{tabular}{lrrrrrrr} 
Bajo & 14 & 9.5 & 18 & 12.2 & 1.0 & - \\
\hline Medio & 104 & 70.7 & 83 & 56.5 & 1.5 & $0.7-3.5$ \\
\hline Alto & 29 & 19.7 & 46 & 31.3 & 0.8 & $0.3-2.0$
\end{tabular}


Continuación...

Índice
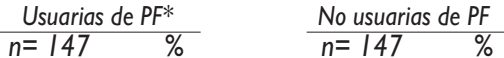

RM Cruda

IC $95 \%$

Rechazo a la violencia

\begin{tabular}{lrrrrrrr} 
Bajo & 9 & 6.1 & 10 & 6.8 & & 1.0 & - \\
\hline Medio & 23 & 15.6 & 16 & 10.9 & 1.6 & $0.5-5.3$ \\
\hline Alto & 115 & 78.2 & 121 & 82.3 & & 1.1 & $0.4-3.1$
\end{tabular}

Equidad de género

\begin{tabular}{|c|c|c|c|c|c|c|}
\hline Bajo & 60 & 40.8 & 57 & 38.8 & 1.0 & - \\
\hline Medio & 68 & 46.3 & 71 & 48.3 & 0.9 & $0.6-1.5$ \\
\hline Alto & 19 & 12.9 & 19 & 12.9 & 1.0 & $0.5-2.0$ \\
\hline
\end{tabular}

Autonomía en relaciones sexuales

\begin{tabular}{|c|c|c|c|c|c|c|}
\hline Bajo & 12 & 8.2 & 21 & 14.3 & 1.0 & - \\
\hline Medio & 20 & 13.6 & 28 & 19.0 & 1.3 & $0.5-3.5$ \\
\hline Alto & 115 & 78.2 & 98 & 66.7 & 2.2 & $1.0-5.0$ \\
\hline
\end{tabular}

*Servicios de planificación familiar

‡ Durante el último año

$\S$ Los totales son de la pregunta anterior: quienes han hablado con su pareja sobre anticoncepción (usuarias I35, no usuarias II0)

Fuente: proyecto:"Género, poder, cultura y salud reproductiva en México", 2006

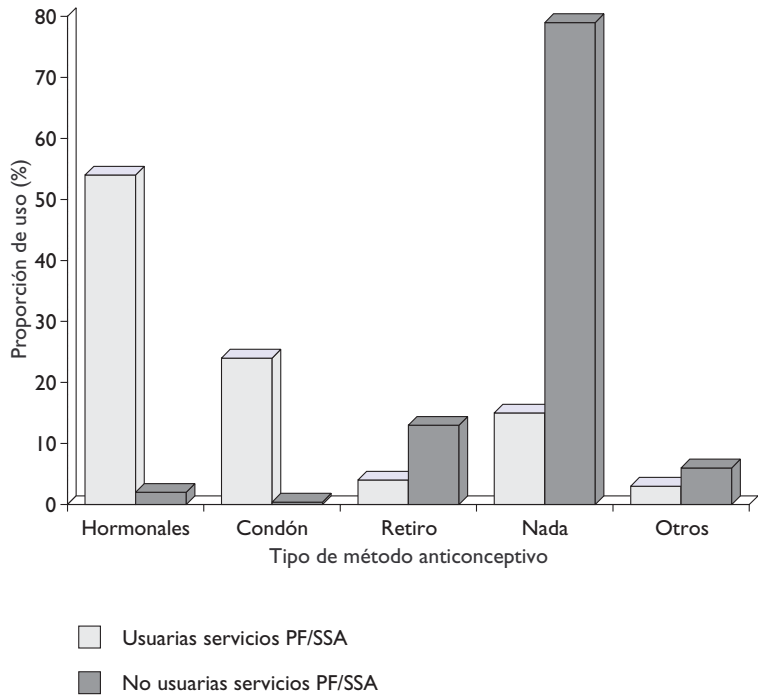

Figura I. Proporción de uso de mÉtodos anticoncepTIVOS EN LA ÚLTIMA RELACIÓN SEXUAL, POR USUARIA Y NO USUARIA DE SERVICIOS DE PLANIFICACIÓN FAMILIAR DE LA SSA. Guanajuato, México, 2003
Al llevar a cabo el análisis multivariado, se encontró una asociación positiva y estadísticamente significativa entre ser usuaria de los servicios de PF de la SSA y la paridad. Así, mujeres que refirieron antecedente de más de seis embarazos en su vida tuvieron más posibilidades de ser usuarias de los servicios de PF, comparadas con aquellas que notificaron de ninguno a dos embarazos en su vida ( $\geq 6$ embarazos: $R M=4.4$; IC95\% 1.4, 13.8). Otras variables asociadas positiva y significativamente con el uso de los servicios de PF de la SSA en Guanajuato, fueron: referir que sí existe comunicación con su pareja sobre temas de PF, ( $R M=3.5$; IC95\% 1.4-9.3); el antecedente de uso de condón masculino con su pareja alguna vez en su vida, $(R M=2.1 ;$ IC95\% 1.0-4.8), y mujeres con mayor puntaje en el índice de poder de decisión (grado alto: $R M=3.2$; ; IC95\% 1.4-7.4 comparado con grado bajo), en donde también se encontró una tendencia positiva significativa (cuadro IV).

Las pruebas de tendencia fueron estadísticamente significativas para los índices de poder de decisión y autonomía en las relaciones sexuales, así como para la variable paridad (cuadro IV). 


\section{Cuadro IV}

Resultados del ANÁLISIS MULTIVARIADO SOBRE CARACTERÍSTICAS ASOCIADAS A LA UTILIZACIÓN DE LOS SERVICIOS DE PLANIFICACIÓN FAMILIAR DE LA SSA, EN EL ÚLTIMO AÑO, POR PARTE DE MUJERES RESIDENTES en Guanajuato, México, 2003

Variables

RMAjustada * IC $95 \%$

Escolaridad (años)

\begin{tabular}{llc} 
Sin educación (cero) & 1.0 & - \\
\hline Primaria ( I-6) & 2.2 & $1.0-5.0$ \\
\hline Secundaria y más (7-+) & 2.2 & $0.8-5.9$
\end{tabular}

Edad inicio vida sexual (años)

\begin{tabular}{lcc}
$\geqq 20$ años & 1.0 & - \\
\hline $15-19$ & 1.1 & $0.6-2.2$ \\
\hline$<15$ & 1.5 & $0.4-6.4$
\end{tabular}

Paridad ${ }^{\ddagger}$

\begin{tabular}{llc}
$0-2$ & 1.0 & - \\
\hline $3-5$ & 1.5 & $0.7-3.0$ \\
\hline 6 y más & 4.4 & $1.4-13.8$
\end{tabular}

Comunicación con su pareja sobre $\mathrm{PF} \S$

\begin{tabular}{ccc} 
No & 1.0 & - \\
\hline Sí & 3.5 & $1.4-9.3$
\end{tabular}

Usó condón masculino alguna vez en su vida

\begin{tabular}{ccc} 
No & 1.0 & - \\
\hline Sí & 2.1 & $1.0-4.8$
\end{tabular}

Poder de decisión $n^{\ddagger}$

\begin{tabular}{llc} 
Bajo & 1.0 & - \\
\hline Medio & 1.6 & $0.8-3.6$ \\
\hline Alto & 3.2 & $1.4-7.4$
\end{tabular}

Rechazo a la violencia

\begin{tabular}{lcc} 
Bajo & 1.0 & - \\
\hline Medio & 1.8 & $0.4-8.3$ \\
\hline Alto & 1.0 & $0.3-3.8$
\end{tabular}

Autonomía en relaciones sexuales ${ }^{\ddagger}$

\begin{tabular}{lcc} 
Bajo & 1.0 & - \\
\hline Medio & 1.4 & $0.4-4.5$ \\
\hline Alto & 2.3 & $0.8-6.7$
\end{tabular}

\footnotetext{
* Razón de momios ajustada por todas las variables del modelo

‡ Prueba de tendencia $p<0.05$

$\S$ Planificación familiar
}

Fuente: proyecto: "Género, poder, cultura y salud reproductiva en México", 2006

\section{Discusión}

La anticoncepción forma parte integral de la salud de mujeres y hombres, y brinda un importante beneficio en la calidad de vida al ofrecer más herramientas para garantizar que la decisión respecto a cuándo comenzar a utilizar un método anticonceptivo, y cuál es el que se adecua mejor a los propósitos reproductivos de cada persona; es una tarea que involucra tanto a las personas interesadas como al Sector Salud.

Los resultados de este estudio reflejan que un porcentaje considerable de aquellas mujeres que no son usuarias de los servicios de PF de la SSA (21\%), también utilizan métodos anticonceptivos. Lo anterior indica que esa población necesita utilizar algún método de regulación de la fecundidad, y que lo obtiene a través de fuentes distintas al Sector Salud. Aquí cabe destacar que los métodos que utiliza ese segmento de la población podrían considerarse como menos efectivos, pues las mujeres refirieron utilizar en mayor proporción métodos naturales, como el retiro, que tiene un nivel menor de eficacia en comparación con los hormonales o con el DIU, utilizados en mayor proporción por las usuarias de los servicios de PF.

Por otro lado, las usuarias mostraron una paridad mayor a las no usuarias, lo que sugiere que estas mujeres llegan a los servicios de PF principalmente para detener la procreación, y no tanto para limitar el número de hijos o para espaciar el periodo entre ellos. Esto podría interpretarse como que el hecho mismo de la procreación les da un mayor acceso a la información sobre la PF y, por tanto, se favorece la decisión de utilizar algún método anticonceptivo. Es decir, para una mujer sin antecedentes de un embarazo es más difícil acudir a un servicio de PF ya que no se "justificaría su acceso".

Los datos anteriores reflejan que, a pesar de que las mujeres participantes en este estudio cuentan con características sociodemográficas similares, la difusión y la accesibilidad de los servicios de PF no están al alcance de todas las mujeres por igual y, por tanto, la información acerca de la PF no siempre llega en el momento que se necesita.

La conducta reproductiva, y por ende la salud reproductiva, se encuentra estrechamente asociada a la condición de género de los individuos. La equidad de género y el empoderamiento sexual y reproductivo son procesos paralelos. En países como México se ha documentado que la desigualdad de género se asocia estrechamente a las dificultades que enfrentan muchas mujeres para apropiarse de su cuerpo, su sexualidad y 
su salud reproductiva. ${ }^{19}$ Por ejemplo, algunos estudios han señalado que las decisiones reproductivas de las mujeres y de los hombres mexicanos, como la edad al primer embarazo, el número de hijos, o el uso de métodos anticonceptivos, están influidas por la inequidad de poder en la sociedad y la familia. ${ }^{20}$ Dicha desigualdad es el resultado histórico de prácticas sociales basadas en una valorización diferencial de las mujeres con respecto a los hombres, y en el concomitante desarrollo de valores y creencias que, a su vez, la refuerzan.

Esta situación ha quedado manifiesta en diversos estudios nacionales e internacionales donde se ha demostrado, entre otras aspectos, que la estructura social de género tiene un impacto en la PF. De igual manera, la comunicación sobre este tema con la pareja, así como el conocimiento que el varón tiene sobre PF es un factor que influye en la decisión del uso de algún método de PF en la pareja. ${ }^{21-25}$ La presente investigación coincide con los hallazgos de esos estudios; así, se encontró que tener comunicación con la pareja en cuanto a los métodos anticonceptivos, y contar con su apoyo para utilizarlos, son variables que están relacionadas con el hecho de acudir a los servicios de PF. Lo anterior conduce, una vez más, a reflexionar sobre la importancia de incluir a los varones en los servicios de PF, ciertamente sin anular la participación de las mujeres en las decisiones reproductivas; el número total de hijos, el espaciamiento entre ellos y el uso de métodos anticonceptivos, son aspectos en los cuales la participación tanto del hombre como de la mujer es esencial.

El poder de decisión y la autonomía de las mujeres representan dos dimensiones intrínsecamente ligadas al empoderamiento femenino. ${ }^{26}$ En este sentido, se han notificado hallazgos sobre el poder de decisión de las mujeres y su autonomía, en relación con el uso de métodos anticonceptivos. Un estudio realizado en México por Casique I., informó que aquellas mujeres que se encuentran en necesidad de anticoncepción (espaciar número de hijos o haber terminado su reproducción de acuerdo con su expectativa reproductiva), tienen mayor posibilidad de ser usuarias de algún método anticonceptivo si es más alto su índice de autonomía y poder de decisión. ${ }^{27}$ Similar hallazgo notificaron Bloom y colaboradores al identificar una asociación significativa entre índice de autonomía de la mujer y uso de servicios de atención prenatal y postnatal. ${ }^{28}$ Los hallazgos anteriores respaldan los resultados del presente trabajo en cuanto a que el poder de decisión de las mujeres se relaciona positiva y significativamente con el uso de servicios de PF.

El grado de autonomía de una persona es inseparable del grupo social al que pertenece. Por tanto, el grado de autonomía que una mujer pueda manifestar dependerá de la autonomía posible de su grupo social y de aquella que las mujeres de la sociedad a la que pertenece hayan alcanzado. En el caso específico de la autonomía de género, lo que se aborda es el grado de libertad que una mujer tiene para poder actuar de acuerdo con su elección y no con la de otros. En tal sentido, hay una estrecha relación entre la adquisición de autonomía de las mujeres y los espacios de poder que puedan instituir, tanto individual como colectivamente. ${ }^{29}$ En cuanto a la autonomía, en este trabajo se analizó únicamente lo que involucra a las relaciones sexuales, es decir, el grado de negociación que tiene la mujer con su pareja referente a las relaciones sexuales, que si bien este es un componente de la autonomía femenina, no envuelve al concepto global o el grado de autonomía en otras áreas de la vida de la mujer.

El hecho de que las mujeres que acuden a los servicios de PF hayan mostrado un nivel de poder de decisión y autonomía en las relaciones sexuales mayor a aquellas que no acuden a los servicios, las coloca en una posición más equitativa en la relación de pareja, al menos en cuanto a la toma de decisiones en el ámbito reproductivo, así como en un mayor control en el bienestar de su salud. Como se ha observado en algunas investigaciones, aquellas parejas donde a las mujeres se les limitan, aunque sea en forma mínima, los valores y las actitudes que tradicionalmente han internalizado desde la niñez participan de manera activa en la toma de decisiones frente a cuántos hijos tener, cuándo y cómo controlar la reproducción. Es decir, se observa un mayor convencimiento femenino del derecho a la igualdad de decisión. ${ }^{30}$

El estudio tiene algunas limitaciones que es necesario considerar; por un lado, se desarrolló con una población específica del estado de Guanajuato, exclusivamente con mujeres de bajo nivel socioeconómico, por lo que es imprescindible tener cautela en la extrapolación de sus resultados. Por el otro lado, al tratarse de un diseño transversal es imposible inferir causalidad entre el grado de poder y autonomía en las relaciones sexuales de las mujeres y su utilización de servicios de PF de la SSA.

En este estudio se empleó como variable dependiente el uso de servicios de PF de la SSA, y no la utilización de métodos de PF. Es importante recalcar que si bien la SSA es un proveedor importante de servicios de salud reproductiva en la población de estudio, actualmente las mujeres obtienen los métodos de PF de otras fuentes, por lo cual es importante, por una parte, investigar más a fondo para conocer cuáles son las barreras tanto individuales como sociales que están impidiendo que aquellas mujeres que desean llevar el control de su fecundidad lleguen a los servicios de PF, y por otra parte, desarrollar estrategias que permitan a las mujeres recibir información sobre PF antes de presentar un evento obstétrico y no después. 
El hecho de haber encontrado relación entre la utilización de servicios de PF de la SSA con dos componentes del empoderamiento femenino, así como con la comunicación con la pareja, apoya la hipótesis de que un mayor empoderamiento de la mujer y una mayor comunicación con su pareja contribuirán a lograr una relación más equitativa. A su vez, esto hará más factible que la toma de decisiones sobre cuestiones reproductivas se lleve a cabo de una forma más informada y compartida. Por lo tanto, el desarrollo de estrategias que involucren a los hombres a la vez que fomenten y fortalezcan el empoderamiento femenino, contribuye a obtener mejores vías para la promoción del uso de servicios de PF.

\section{Agradecimientos}

Se agradece al personal del Centro de Atención al Adolescente, CASA, por su participación en el levantamiento de la información, en los municipios de San Miguel de Allende y San Luis de la Paz, Guanajuato. Asimismo, se agradece a las doctoras Maya Blum y Cynthia Harper, del Centro de Investigaciones en Salud Reproductiva, de la Universidad de California, San Francisco, EUA, su contribución al análisis estadístico de la información, y a la doctora Irene Casique, del Centro Regional de Investigaciones Multidisciplinarias de la UNAM, su asesoría en la construcción de los índices de empoderamiento.

\section{Referencias}

I. Population Reference Bureau. Family planning saves lives. $2^{\mathrm{a}}$. edición. Washington, DC: PRB, 1991.

2. Organización Mundial de la Salud. Comunicación sobre planificación familiar en el marco de la salud reproductiva. Ginebra: OMS, 1997.

3. Consejo Nacional de Población. Indicadores de Salud Reproductiva en la República Mexicana. [Consultado el 7 de noviembre de 2006]. Disponible en: http://www.conapo.gob.mx/00cifras/00salud.htm. 4. International Planned Parenthood Federation. Country Profile, Mexico. [Consultado el I 3 de septiembre de 2003]. Disponible en: http://ippf. org/;ub/IPPF_regions.

5. Langer-Glas A. Embarazo no deseado y el aborto inseguro: su impacto sobre la salud en México. Gac Med Mex 2003; 139: S3-S7.

6. Organización Panamericana de Salud. Política de la Organización Panamericana de la Salud en materia de igualdad de género. Rev Panam Salud Publica 2006; 19:137-140.

7. Mason $\mathrm{K}$, Smith $\mathrm{H}$. Husbands versus wives fertility goals and use of contraception: The influence of gender context in five Asian countries. Demography 2000; 37: 299-3II.

8. Govindasamy P,Anju M. Women's position and family planning in Egypt. Stud Fam Plann 1996; 27: 328-340.

9. Hogan D, Betemariam B, Assefa H. Household organization, women's autonomy, and contraceptive behavior in southern Ethiopia. Stud Fam Plann 1999; 30: 302-314.
10. Pulerwitz J, Gortmaker S, De Jong W. Measuring sexual relationship power in HIV/STD research. Sex Roles 2000; 42: 637-660.

II. Steenbeek A. Empowering health promotion: a holistic approach in preventing sexually transmitted infections among first nations and inuit adolescents in Canada.J Holist Nurs 2004; 22:254-266.

12. Ollila E, Koivusalo M, Hemminki E. International actors and population policies in India, with special reference to contraceptive policies. Int J Health Serv 2000; 30: 87-II0.

13. Casique I. Empoderamiento femenino y uso de anticonceptivos en México. Boletín Género y Salud en Cifras 2003; 1:13-18.

14. Blanc A. The effect of power in sexual relationships on sexual and reproductive health:An examination of the evidence. Stud Fam Plann 2001; 32: 189-2।3.

15. Blanc A, Wolff B. Gender and decision-making over condom use in two districts in Uganda. Afr J Reprod Health 200I; 5: 15-28.

16. Ayaga A. Spousal communication and family planning behavior in Navrongo:A longitudinal assessment. Stud Fam Plann 2002; 33: 185-194. 17. Instituto Nacional de Estadística, Geografía e Informática. Síntesis metodológica del XII Censo General de Población y Vivienda 2000. [Consultado el 12 de noviembre de 2006]. Disponible en: http://www. inegi.gob.mx/est/default.asp? $c=2343$

18. Secretaría de Salud del Estado de Guanajuato. Plan Estatal Maestro en Infraestructura en Salud para Población Abierta del 2000 PEMISPA y Registros para la infraestructura en Salud (Renis), 200I. [Consultado el 20 de agostode 2005]. Disponible en: http://www.guanajuato.gob.mx/ssg/ infraestructura/index.htm

19. Amuchastegui A. Dialogue and the negotiation of meaning: constructions of virginity in Mexico. Cult Health Sex 1999; I(I):79-93. 20. Szasz I. Pensando en la salud reproductiva de hombres y mujeres. En: Bronfman M, Denman C. Salud reproductiva:Temas y debates. México: Instituto Nacional de Salud Pública, 2003.

21. Pulerwitz J,Amaro H, De Jong W, Gortmaker SL, Rudd R. Relationship power, condom use and HIV risk among women in the USA.AIDS Care 2002; 14:789-800.

22. Sternberg P, Hubley J. Evaluating men's involvement as strategy in sexual and reproductive health promotion. Health Promot Int 2004; 19:389-396.

23. Ikechebelu J, Joe-lkechebelu N, Obiajulu F. Knowledge, attitude and practice of family planning among lgbo women of south-eastern Nigeria.J Obstet Gynaecol 2005; 25:792-795

24. Wolff B, Blanc A, Ssekamatte-Ssebuliba J.The role of couple negotiation in unmet need for contraception and the decision to stop childbearing in Uganda. Stud Fam Plann 2000; 3I: I24-I 37.

25. Roja LR. La participación de los varones en los procesos reproductivos: un estudio cualitativo en dos sectores sociales y dos generaciones en la ciudad de México. Papeles Poblac 2002; 31: 189-2I7.

26. Casique I.Trabajo femenino, empoderamiento y bienestar de la familia. En: Latin American Studies Association, LASA; 2003 Marzo 27-29; Dallas (TX), EUA.

27. Casique I. Uso de anticonceptivos en México: ¿Qué diferencia hacen el poder de decisión y la autonomía femenina? Papeles Poblac 2003; 35 : 209-236.

28. Bloom S, Wypij D, Das Gupta M. Dimensions of women's autonomy and the influence on maternal health care utilization in a North Indian City. Demography 200I; 38:67-78.

29. Organización de las Naciones Unidas.Autonomía de las mujeres e igualdad de género. En: Objetivos de desarrollo del milenio: Una mirada desde América Latina y el Caribe. Santiago de Chile: Naciones Unidas, 2005: III-140.

30. Batliwala S. El significado del empoderamiento de las mujeres: nuevos conceptos desde la acción. En: León M. Poder y empoderamiento de las mujeres. Colombia:TM Editores, 1998: 187-21 I. 